\title{
Altered Excitability of the Crayfish Lateral Giant Escape Reflex during Agonistic Encounters
}

\author{
Franklin B. Krasne, Ashkan Shamsian, and Raghavendra Kulkarni \\ Department of Psychology and Brain Research Institute, University of California at Los Angeles, Los Angeles, \\ California 90095-1563
}

\begin{abstract}
The excitability of the lateral giant escape reflex of socially dominant and submissive crayfish at rest and during agonistic encounters was studied and compared. During agonistic encounters the excitability of the lateral giant reflex falls, substantially in subordinates and slightly in dominants, whereas at rest excitability seems to be independent of social status. Thus, paradoxically, socially dominant animals are more likely to execute lateral giant escape reactions during interactions than are subordinates. It is suggested that subordinates under threat of attack tend to engage circuitry involved in flexible, nonreflex ("voluntary") types of escape not mediated by giant neurons and therefore inhibit giant neuron-mediated reflex circuitry that produces prompt, but less adaptive, responses. In contrast,
\end{abstract}

dominants go about their business, mainly ignoring their conspecifics and relying on reflex escape to protect them from unexpected attack. Consistent with this view, escape of subordinates during agonistic encounters is mediated by nongiant, not reflex, circuitry. These observations and their interpretation suggest a possible functional role for recently described social status-dependent serotonergic modulation of the lateral giant reflex, which is inhibitory in sign in subordinates and facilitatory in dominants.

Key words: 5-HT; serotonin; agonistic behavior; lateral giant; social dominance; aggression; crayfish; escape reflex; reflex modulation
Escape responses mediated by the lateral giant (LG) axons of the crayfish are known to be subject to modulation under a number of behavioral circumstances. Lateral giant escape is inhibited when an animal is restrained (Krasne and Wine, 1975), feeding (Krasne and Lee, 1988), exhibiting defensive posture (Beall et al., 1990), walking backward (Beall et al., 1990), or is in the process of executing a previously begun escape response (Roberts, 1968); inhibition also partially mediates habituation to repetitive stimulation (Krasne and Teshiba, 1995). The reflex is facilitated when an animal has been traumatized by strong stimulation (Krasne and Glanzman, 1986). Investigation of the mechanisms of several of these forms of modulation has provided insights of rather general neurobiological interest (Krasne and Bryan, 1973; Krasne and Lee, 1988; Vu et al., 1993; Krasne and Teshiba, 1995).

For those kinds of inhibition in which the matter has been studied, available evidence suggests that GABA, acting at several different loci on the LG dendrites and also in some cases at previous points in the circuit, is the inhibitory transmitter (Roberts, 1968; Kennedy et al., 1980; Vu and Krasne, 1993; Krasne and Teshiba, 1995). Serotonin is known to produce inhibition with properties quite similar to some types of GABA-ergic inhibition that have been studied, but no actual role for serotonergic modulation has been found (Glanzman and Krasne, 1983; Vu and Krasne, 1993).

However, Yeh et al. (1996, 1997) recently reported that the sign of the modulatory effect of serotonin on the LGs is dependent on an animal's social status. In subordinates serotonin inhibits es-

Received July 22, 1996; revised Oct. 3, 1996; accepted Nov. 7, 1996.

This research was supported by National Institutes of Health Grant NS8108. We thank Terri Teshiba for her technical help and Donald Edwards for reading a version of this manuscript.

Correspondence should be addressed to Dr. Krasne at the above address.

Copyright (C) 1997 Society for Neuroscience 0270-6474/97/170709-08\$05.00/0 cape, apparently as was seen for animals of unspecified social status by Glanzman and Krasne (1983), whereas in dominants, and also in socially isolated crayfish, serotonin facilitates LG escape. This rather remarkable social dependency of the modulatory role of serotonin obviously suggests that serotonergic modulation of escape may occur during agonistic interactions. That escape reflex excitability might be modulated during social interactions seems intuitively plausible, but despite its ethological interest this possibility has not, until now, been investigated.

Tail flip escape responses in crayfish can be produced by three different systems (Wine and Krasne, 1972, 1982): (1) circuitry that produces stereotyped, very short-latency upward-directed responses to abdominal mechanosensory stimulation contingent on firing of LG command neurons, (2) circuitry that produces stereotyped, short-latency backward-directed responses to rostral mechanosensory and visual stimuli contingent on the firing of medial giant command neurons, and (3) circuitry that produces nonstereotyped visually guided tail flip swimming responses of rather long and variable latency without the aid of giant neurons. We refer here to the stereotyped giant fiber-mediated responses as "reflex" to contrast them with the more flexible, long-latency nongiant or "voluntary" [Wine and Krasne (1972) and Krasne and Wine (1984) discuss the rationale for this terminology] responses. Most of the observations reported here concerned the LG reflex.

\section{MATERIALS AND METHODS}

Animals and maintenance. Male Procambarus clarkii, $\sim 8-10 \mathrm{~cm}$ in length (rostrum to telson) were obtained from various local suppliers. They were maintained and tested as pairs in aerated, filtered 5 gallon aquaria separated, except during social testing, by a porous partition. Their claws were banded to protect electrode leads, but this seemed to have little effect on their agonistic behavior.

Preparation. Before surgery animals were allowed to interact for $\sim 5$ min, which was long enough to determine which dominated, as determined by chase, attack, and threat by the dominant and evasion or 


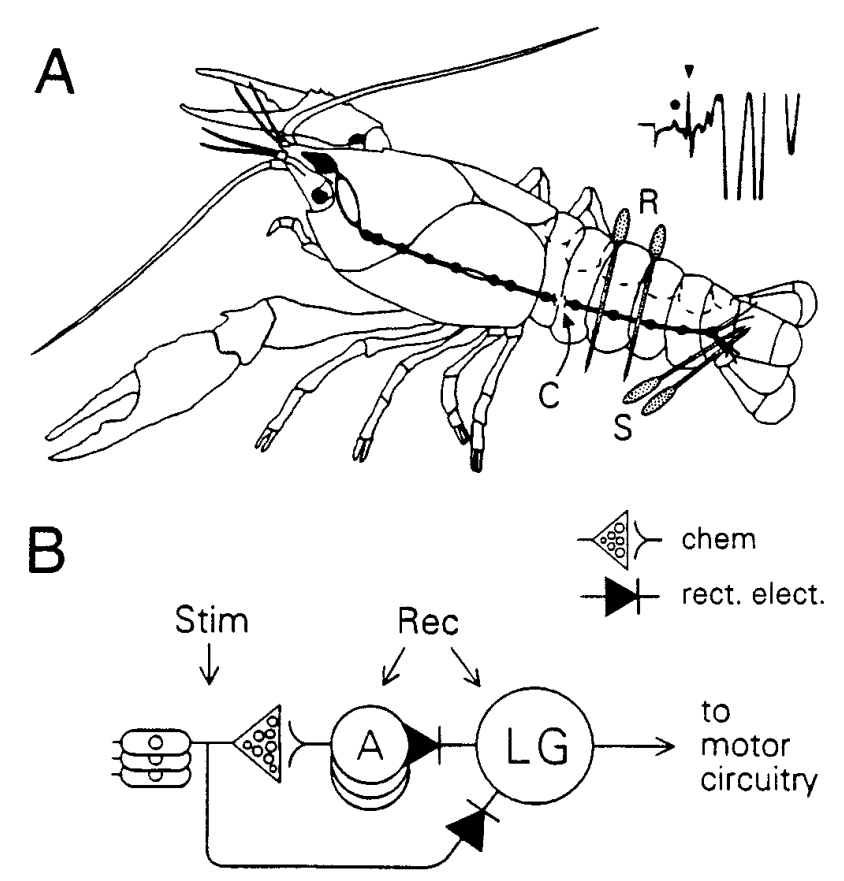

Figure 1. Chronic preparation. $A$, Recording $(R)$ and stimulating $(S)$ electrodes were implanted as indicated. Recording electrodes were placed dorsal to the cord $(C)$, where they detected the firing of int $\mathrm{A}$ as small spikes (dot in inset) and LG firing as large spikes (triangle in inset). The stimulating electrodes lie over afferent-containing roots; the number of fibers that fire increases as a function of stimulus intensity. $B$, Circuit of afferent portions of LG reflex indicating parts of circuit activated by stimulating electrodes (Stim) and detected by recording electrodes (Rec).

submissive posture of the subordinate. Work by others has shown relative social status to be quite stable over time (Bovbjerg, 1953; Lowe, 1956). This has been our experience, as well; only one pair reversed its dominance relationship after implantation (for analysis, this pair was classified in terms of its postimplantation status).

Animals were implanted with stimulating electrodes on roots 2-4 of one side of the last abdominal ganglion, and recording electrodes were placed dorsal to the ventral nerve cord on the 2-3 and 3-4 abdominal connectives (Fig. 1; for details, see Glanzman and Krasne, 1983). Electrodes were stainless steel 00 insect pins (Wards) insulated except for a small gap where the electrodes crossed the nerve fibers to be recorded or stimulated. The stimulating electrodes were used to test the excitability of the LG reflex, and the recording electrodes were used to monitor firing of the LGs and also the largest of the first-order sensory interneurons (int A) intercalated between sensory neurons and LGs (Fig. 1). Then 1-3 d (usually $2 \mathrm{~d}$ ) were allowed for recovery from the implantation before testing.

Testing. Each pair was tested on three occasions, always separated by at least $1 \mathrm{~d}$. During all testing single $0.2 \mathrm{msec}$ test pulses to sensory root electrodes were applied every $1.5 \mathrm{~min}$; a computer varied the pulse voltage from trial to trial to evaluate continuously, in alternation, the thresholds for firing of the LGs and of int A (Fig. 2). Although LG firings were scored for convenience, it is firmly established that short-latency upward tail flips occur if and only if the LGs fire (Wine and Krasne, 1972; Olson and Krasne, 1981; Krasne, unpublished data); thus, it can be assumed that when the LGs fired, LG-type behavioral responses always occurred. Stimuli were controlled by a D/A converter with a $1 \mu \mathrm{A}$ step size; stimulus levels are presented as the number of steps above 0 . Both animals were stimulated approximately in synchrony (test stimuli separated by $\sim 1 / 4 \mathrm{sec}$ - the time needed for the computer to acquire and process electrophysiological data).

Before each session, the approximate threshold of int A for each animal was determined by trial and error. Then the initial stimulus level for int A was set just above threshold and that for LG at three times that level (stimuli were kept within 8 times the int A threshold). Thereafter, the threshold-tracking algorithm increased stimulus levels whenever the unit under examination failed to fire to the preceding stimulation and

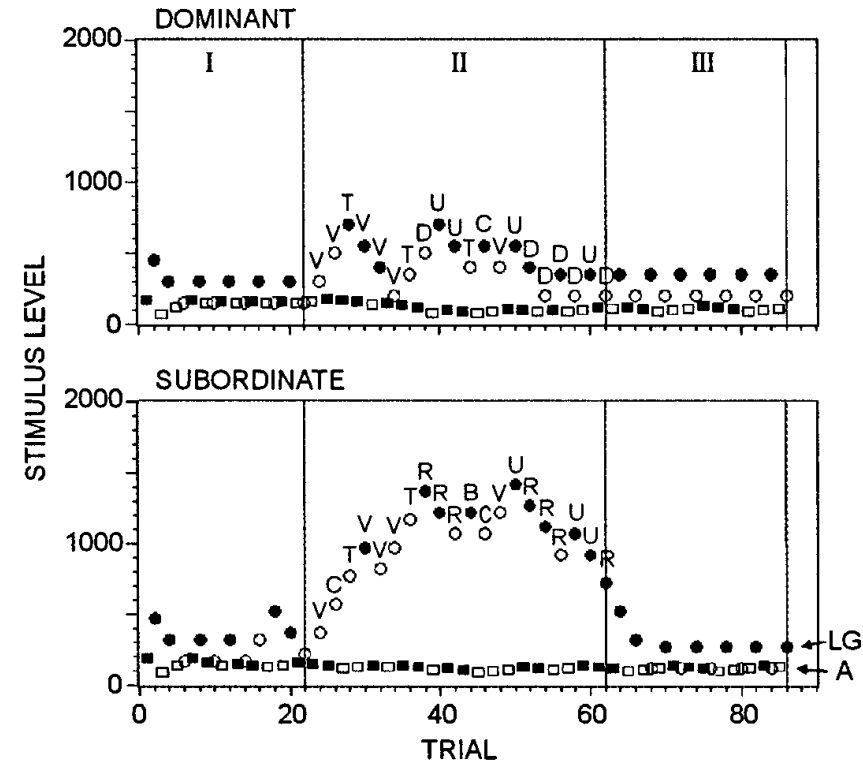

Figure 2. Results from a typical experimental session. Each point shows the stimulus level applied on a trial (stimulus scale arbitrary). Filled symbols indicate that the LGs (circles) or interneuron A (squares) fired, and open symbols indicate that they did not. Animals were separated during periods I and III and were together during period II. Letters indicate what the animal was doing at the time each stimulus was given during period II (see Table 1 for codes).

reduced it when the unit did fire. When on adjacent trials a response occurred to the greater, but not the lesser, stimulus, the two stimulus levels were saved as "upper" and "lower bounds" on the threshold, and the step by which subsequent stimulus levels were changed was halved (down to a minimum step size); when responding to stimuli was not predicted by current estimates of the upper and lower bounds on threshold, step size was doubled (up to a maximum step size).

After electrode leads were attached, $30 \mathrm{~min}$ was allowed for animals to recover from the disturbance. Thresholds were tracked for $\sim 30 \mathrm{~min}$ before bringing the animals together (period I), for $60 \mathrm{~min}$ with the animals together (period II), and for $30 \mathrm{~min}$ with them again separated (period III). Animals were brought together for interaction either by removing the partition separating them or by lifting both animals and placing them on the dominant's side of the tank; we saw no differences between the results of these procedures. All animals also received control tests in which they were either left undisturbed, except for the test shocks, or were exchanged to their partner's side of the tank during the time corresponding to period II of experimental sessions. Throughout encounters the ongoing behavior of each animal immediately before the occurrence of test shocks was scored according to the code in Table 1.

Data analysis. When on adjacent trials during threshold tracking a response occurred to a greater, but not to a lesser, stimulus, threshold was

\section{Table 1. Behavior scoring codes}

\section{$\mathrm{V}$, Vigorous claw grappling}

C, Claw grappling

A, Approaching other animal

T, Touching other animal

D, Dominant posture (raised open claws, standing high, center of tank, facing other animal)

$\mathrm{R}$, Retreating from other animal

$\mathrm{S}$, Submissive posture (facing away from other animal, at margin of

tank, thorax close to substrate, claws touching substrate)

$\mathrm{B}$, Being touched

U, Unspecified definite movement

Q, Quiescent (not moving, no recognized agonistic posture)

$\mathrm{G}$, Gravel manipulation*

F, Flipping*

*Rarely seen just preceding stimulations and not included in Figures 6-8. 

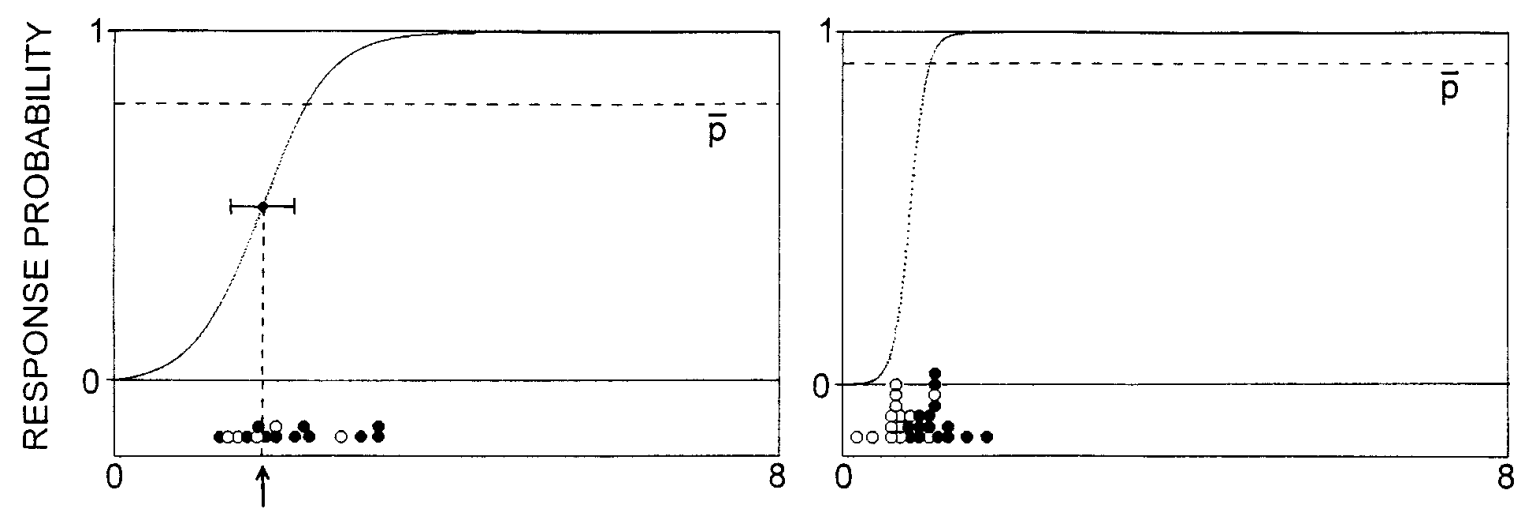

NORMALIZED STIMULUS INTENSITY

Figure 3. Measures of threshold and response probability illustrated. Each panel shows pooled data from several sessions on a single animal. The left panel shows period II trials during which a dominant animal was still but maintaining a dominant posture; the right panel show all trials during period I of the same sessions. At the bottom of each panel is a scatter plot of stimulus levels presented, with those that caused responses shown as filled symbols. Stimulus levels are normalized to the baseline threshold for the session in which they were presented (i.e., they were divided by the average of the threshold voltage during periods I and III for the session). At the top of each panel is shown the best-fitting logistic curve of probability of response as a function of normalized stimulus strength for the data below (see text). The marker at probability 0.5 on the left panel indicates the maximum likelihood values of $T \pm S$. The mean of the curve over normalized stimulus strengths $0-8$ is shown as dashed lines.

assumed to have been crossed, and its value was taken as halfway between the two stimuli. Such estimates from threshold crossings were averaged over periods of the experiment to obtain the estimates of LG thresholds used for data analyses.

For several analyses we wished to make quantitative comparisons between the excitabilities of the escape reflex during different ongoing activities; if tests had been done with the same set of stimulus levels during each type of activity, a direct comparison could have been made of the proportion of positive responses during each condition. However, because the ongoing behavior of the animal could not be controlled, arranging such test conditions would have been next to impossible. Therefore we were faced with data like that at the bottom of Figure 3 , which shows the responses and failures (as filled and open circles, respectively) as a function of stimulus strength in an animal under two conditions. Although it seems obvious that excitability of escape was less under the condition yielding the left-hand set of trials than for those on the right, quantification of this presents a problem. The procedure that we adopted was to assume that probability of response as a function of stimulus strength could be approximated by the sigmoid-shaped logistic function, $p=1 /\left(1+\mathrm{e}^{-(\mathrm{x}-\mathrm{T}) / \mathrm{s}}\right)$. The variable $x$ is a measure of stimulus strength, the parameter $T$ (for threshold), is the stimulus value at which probability of response is 0.5 , and the parameter $S$ (for spread) reflects the degree of gradualness of the rise of the function. The best-fitting logistic function was determined for each set of points, as shown in Figure 3 . The average probability of response across the full range of stimuli (indicted by the dashed lines) then could be taken as a measure of the excitability of the escape reflex that could be expected to be reasonably independent of the particular stimulus values used for testing.

The best-fitting logistic function was determined by making maximum likelihood estimates of the parameters $T$ and $S$. The probability of getting a given set of data was calculated for a range of values of $T$ and $S$, and the values that maximized the probability of the observed data were used as the logistic function parameters; the parameter space was searched with $T$ and $S$ increments of 2 and $1.25 \%$, respectively, of the full range of stimulus values. Although we never actually tested with zero strength stimuli in our experiments, the probability of a spontaneous flip at any arbitrarily chosen moment (i.e., a flip at zero stimulus strength) was virtually nil; therefore, we added to each data set 30 nonresponse trials to constrain the logistic functions to near zero at zero stimulus strength (30 trials being the minimum that produced effective anchoring).

Excitability of the escape reflex tended to be reduced during periods of interaction; thus, it was useful to create a statistic that reflected degree of suppression. The average of arc sin mean probability across periods I and III minus average arc sin mean probability for period II, referred to as the "suppression index," served this purpose. Arc sin transforms of probability were used to make the probabilities, which are constrained between zero and one, more normally distributed. On occasion, a range of $T$ and $S$ values provided equally good fits to the data; then the values that gave the lowest suppression index were used.
Statistical analyses. Wilcoxon signed ranks tests were used to evaluate within-subject differences between treatments and Mann-Whitney $U$ tests to evaluate between-group differences. All tests were two-tailed. When we desired to evaluate the effect of social interaction on escape threshold, we attempted to factor out most of the effect of any gradual shifts of threshold that may have developed because of testing, per se, by calculating the difference in mean threshold between period II and the averages of periods I and the last half of period III (by which time the effects of the previous interaction period seemed to have worn off); we refer to this measure as the period II threshold increase.

\section{RESULTS}

\section{Baseline LG escape reflex excitability of dominants and subordinates does not differ}

In all test sessions the thresholds for LG and int A were determined before bringing the animals together for interaction. In most cases, both for dominants and subordinates, the threshold of LG was close to twice that of int A; however, in two subordinates it was much higher than this (Fig. 4). Although there is certainly no statistically significant difference here $(p>0.2)$, these results raise the possibility that some differences in baseline excitability might be found with further investigation.

\section{Lateral giant escape is depressed during agonistic encounters and more so in subordinates than dominants}

Figure 2 shows the experimental results for a dominant/submissive pair. The stimulus threshold for firing of the giants, and for a corresponding tail flip response, rises during the period of interaction in both animals, although considerably more in the sub-

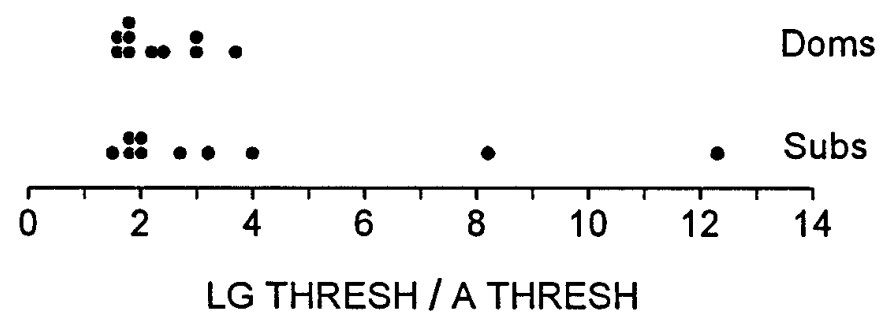

Figure 4. Resting excitability of LG reflex. Scatter plot of ratios of LG to interneuron A threshold are averaged over all periods I for each animal. 

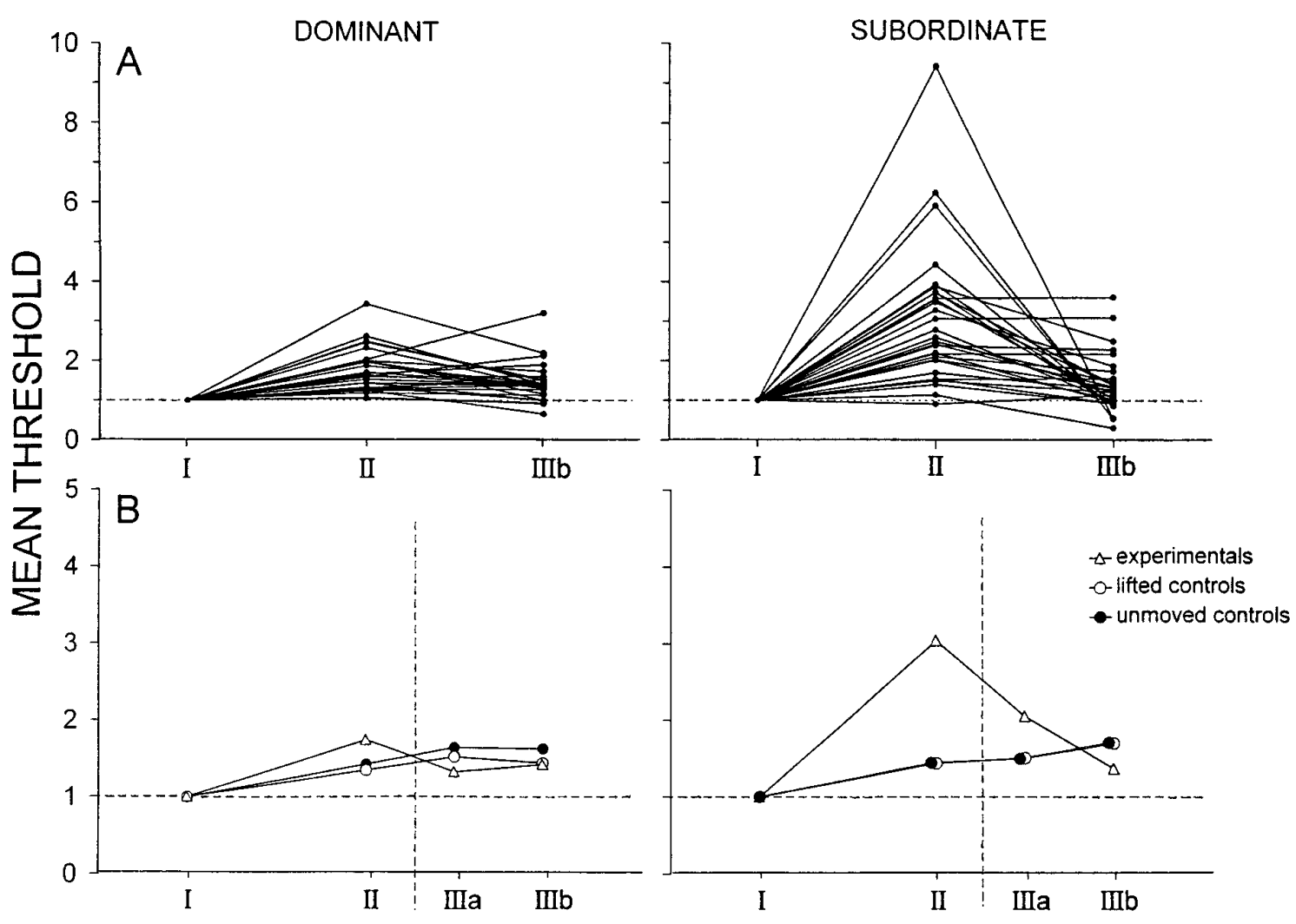

Figure 5. LG thresholds of dominants and subordinates during encounters. For each period, LG thresholds, taken as averages of estimates from individual threshold crossings (see Data Analysis), were normalized to period $I$ values. $A$, All experimental sessions; thresholds for each session are joined by a line. Period $I I I$ was divided into halves (a and b); for clarity only, IIIb values are shown here. $B$, Data averaged across animals. Data for control (see Materials and Methods) as well as for experimental sessions are shown.

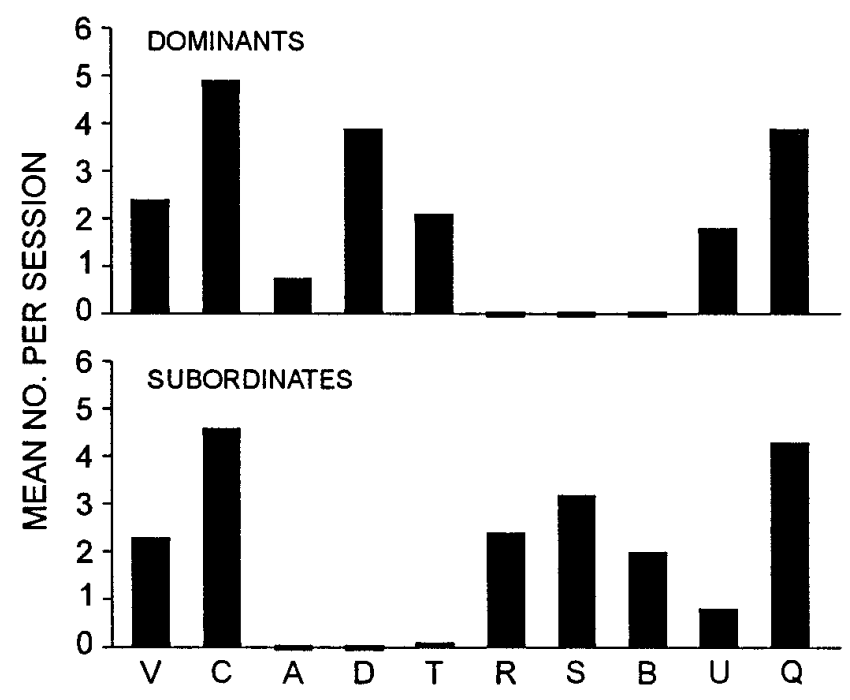

Figure 6. Behavioral differences between dominants and subordinates. The mean number of occurrences per session during period II is shown for each category of behavior.

missive than the dominant, and returns to near baseline when the animals are separated. Figure 5, $A$ and $B$, which summarizes data for all the tests done, shows this pattern to be common, although not without exception. Period II threshold increases (see Materials and Methods) were statistically reliable for both dominants ( $p<$ $0.01)$ and submissives $(p<0.01)$, and increases were significantly greater for subordinates than dominants $(p<0.02)$.
The same animals also were tested without a period of social interaction, either being undisturbed or being handled and placed on their partner's side during the period when in experimental tests they would have been together (Fig. $5 B$ ). There was no detectable difference between the two sorts of control, so the data were pooled. The control animals showed a mild rise in threshold over the course of each session because of habituation; because the rise was a little greater in the earlier part of each session, mean thresholds during period II were slightly greater than averaged period I and IIIb thresholds, with the result that there was a slight period II threshold increase (significant for dominants, but not for submissives). However, the magnitude of this increase was trivial when compared with the increase seen when animals were placed together (in both dominants and submissives, $p<0.01$ for the difference in period II threshold increase between "together" and control sessions).

When animals were separated (period III), escape thresholds fell toward the preinteraction (period I) level, although there seems to have been a tendency for thresholds of dominants to fall a little below those for control runs and for thresholds of subordinates to be elevated relative to control. These differences were not statistically significant, but they warrant further examination (see Discussion). It is difficult to evaluate precisely how rapidly threshold began to fall after separation of the animals, because the computer algorithm used for tracking thresholds had a certain amount of inertia built into it; thus, in animals whose threshold had risen during interaction, it usually did not test with low stimulus levels immediately after separation. However, in eight sessions the stimulus given at 3 min after separation was $>25 \%$ 


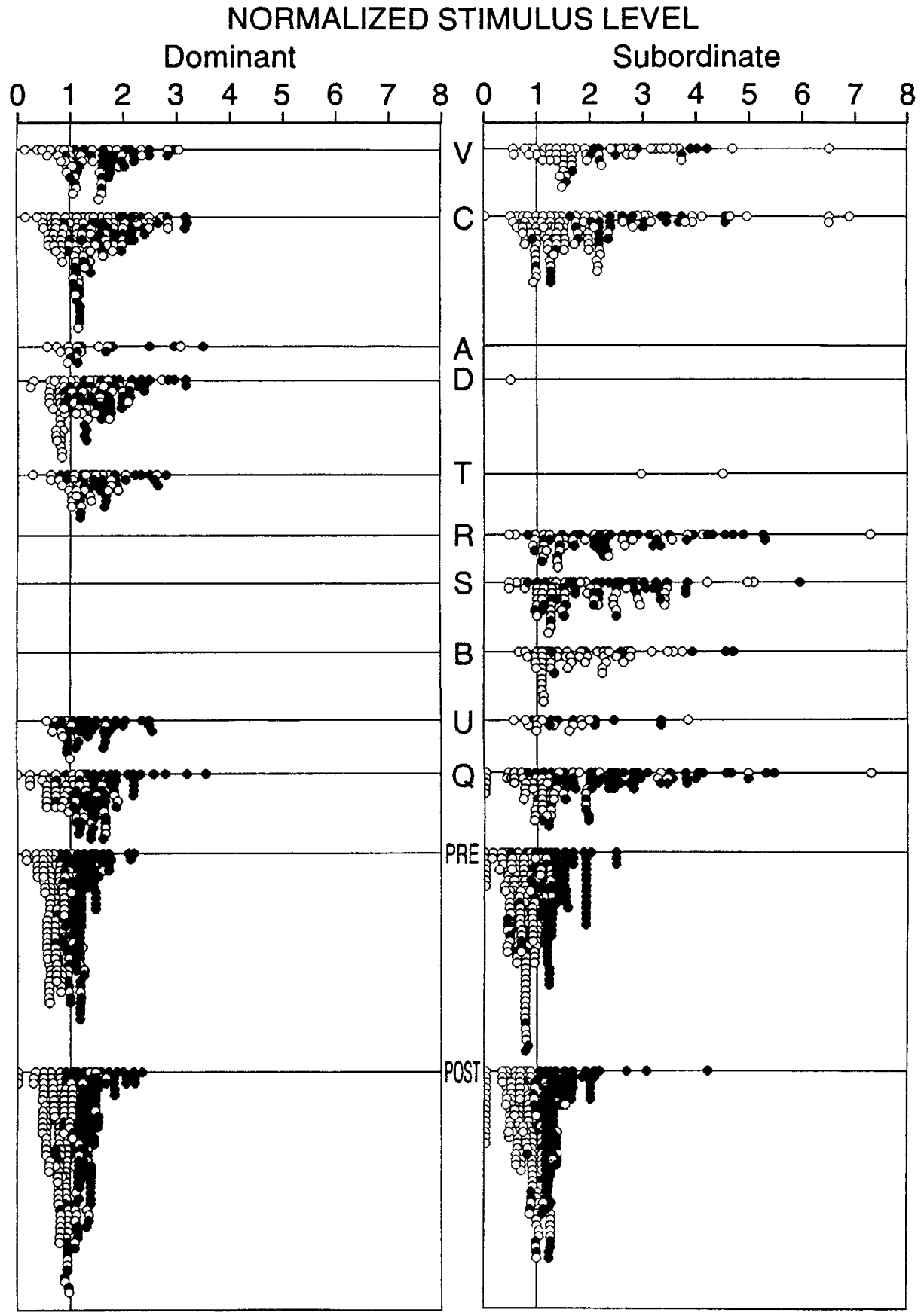

Figure 7. LG reflex excitability associated with different behaviors. For this figure, data from all animals were pooled, and stimulus levels from an encounter were normalized to the average of periods I and III for that encounter. below the difference between the threshold at the end of the interaction period and the preinteraction threshold, and in eight cases it was $>67 \%$ below at 6 min after separation; in each of these cases the stimulus caused a response. Thus, the thresholdelevating effects of interaction seem to wear off quite rapidly once the animals are separated.

\section{Sensory interneuron thresholds remain constant while LG thresholds are elevated}

The threshold of the largest of the interneurons intercalated between sensory neurons and LGs shows no sign of increasing during period II in either the session illustrated in Figure 2 or in any other test session of the study. Thus, insofar as the behavior of interneuron A is representative of that of sensory interneurons in general, the modulation of escape during agonistic encounters seems to be attributable to modulation focused at the level of the LGs.

\section{LG escape is suppressed more during some activities than others}

The activities of animals in each group during formal experimental sessions are consistent with our initial evaluation of their social status (Fig. 6). Dominants approach their conspecifics, whereas subordinates never do so; subordinates retreat from their partners, but dominants do not. Dominants touch subordinates, but subordinates never touch dominants except as part of clawgrappling episodes, which (from informal observation rather than formal behavior ratings) typically are initiated by the dominant, rather than by the subordinate.

Figure 7 displays the results of all test trials run, pooled across 


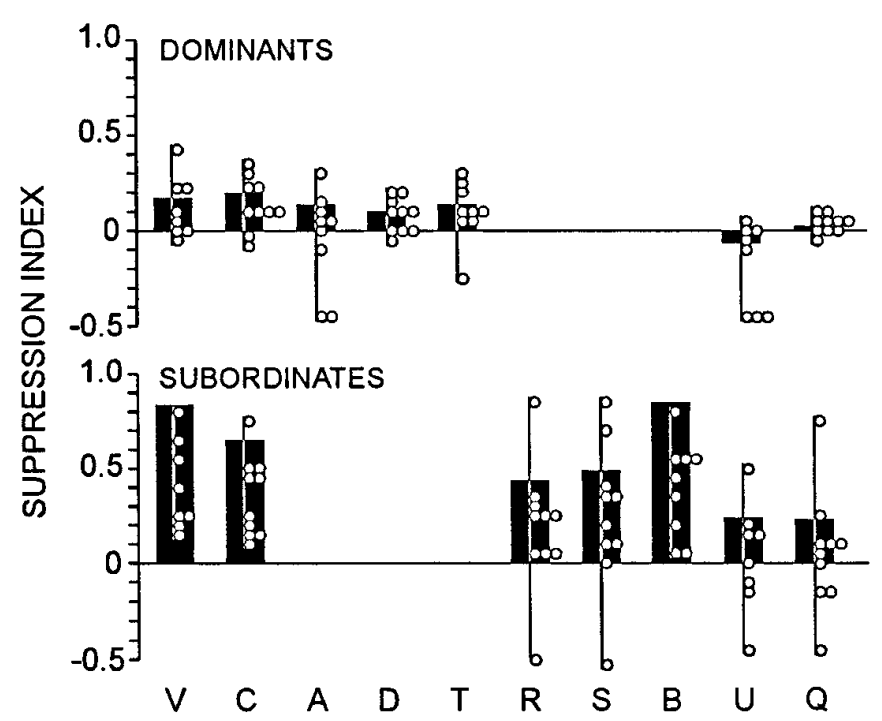

Figure 8. Degree of suppression during specific behaviors. Black bars show suppression index for behaviors, the codes of which are indicated (see Table 1) and calculated from pooled data of Figure 7; open circles show suppression indices for individual animals.

animals but segregated with respect to the kind of activity in which the animal was engaging at the time that reflex test shocks were applied. It seems clear from this chart that during certain activities, such as "vigorous" or "ordinary" claw grappling (categories V and $\mathrm{C}$, respectively) by submissives, escape is suppressed relative to control levels (PRE and POST), whereas suppression of escape is less conspicuous during other behaviors, such as retreat $(R)$ by subordinates or display of "dominant postures" (D) by dominants.

To quantify the differences that seem apparent in Figure 7, we calculated a suppression index for each of the data sets (Fig. 8, black bars; see Materials and Methods). We also did a similar computation for the data of each individual animal, the results of which also appear in Figure 8 (open circles). From these calculated indices it seems that (1) the overall greater tendency of submissives than of dominants to suppress is not simply a product of more engagement by submissives in suppression-associated activities, because even for activity categories that occur in both types of animal (categories V, C, U, and Q) the suppression index, averaged over these categories, is greater in submissives than in dominants $(p<0.02)$. (2) Suppression of escape is not attributable to the animals being together, per se, because when animals are quiescent (category Q), there is relatively little consistent indication of suppression; when animals are pooled across groups, the suppression index is not significantly different from zero $(p \gg$ $0.05)$ and is reliably less than during claw grappling $(p<0.001$ for $\mathrm{Q}$ vs mean for $\mathrm{C}$ and V). (3) Activity, per se, is not responsible for suppression, because there is little suppression during unspecified, but definite, active movements (category $\mathrm{U}$ ); when animals are pooled across groups, the suppression index for $U$ is not significantly different from zero $(p \gg 0.05)$, is reliably less than the mean for activities $\mathrm{C}$ and $\mathrm{V}(p<0.001)$, and is not greater than that for the quiescent condition $(p>0.05)$.

\section{Suppression of the LG escape reflex is variable from moment to moment even during a given activity}

Compared with control periods (I and III), during which there was usually a rather sharp and stable stimulus threshold below which LGs very rarely fired and above which they almost always did (Fig. 7), thresholds during agonistic encounters were erratic. They varied markedly from moment to moment within a test session according to the particular type of activity in which an animal was engaged (Figs. 7, 8). They also varied from time to time even during the occurrence of a given category of behavior. This is illustrated for data from two individual sessions in Figure 9. Whereas threshold during control periods in Figure $9 A, B$ was sharp, it was not so during encounters for the categories of behavior illustrated; thus, in $A$ the threshold seems to vary between somewhere below 700 (arrow 1) to $\sim 1100$ (arrow 2) and in $B$ from $\sim 300$ (arrow 1) to somewhere above 1100 (arrow 2).

The lack of a sharp threshold during agonistic interactions can be seen quantitatively from analysis of the "spread" parameter $(S)$ of the logistic functions fit to the data (see Materials and Methods). During control periods the mean value of $S$ (using normalized stimulus strengths) was 0.09 ; this means that the probability of response went from 0.27 to 0.73 as stimulus strength went from 0.09 below threshold to 0.09 above threshold (threshold here being unity, because stimulus strengths were normalized to control period thresholds). By contrast, the value of $S$ averaged across animals and across each of the types of activities scored during encounters was 0.32 , an approximately three times rise $(p<0.01)$. Because the higher values of $S$ during encounters might have been an artifact of thresholds being higher during agonistic behavior than during periods I and III, we repeated this analysis using the ratio of spread to threshold within each type of activity; $S / T$ rose from 0.11 during control periods to 0.17 averaged across the activity types scored, a $64 \%$ increase $(p<0.05)$.
Figure 9. Variability of LG reflex thresholds during periods of suppression from single sessions on subordinates. Raw stimulus strengths, rather than normalized values, are shown. During pre and post tests threshold was well defined, but during $R$ and $C$ activities thresholds were erratic. During each test thresholds seemed to vary between the levels marked as 1 and 2 (arrows).

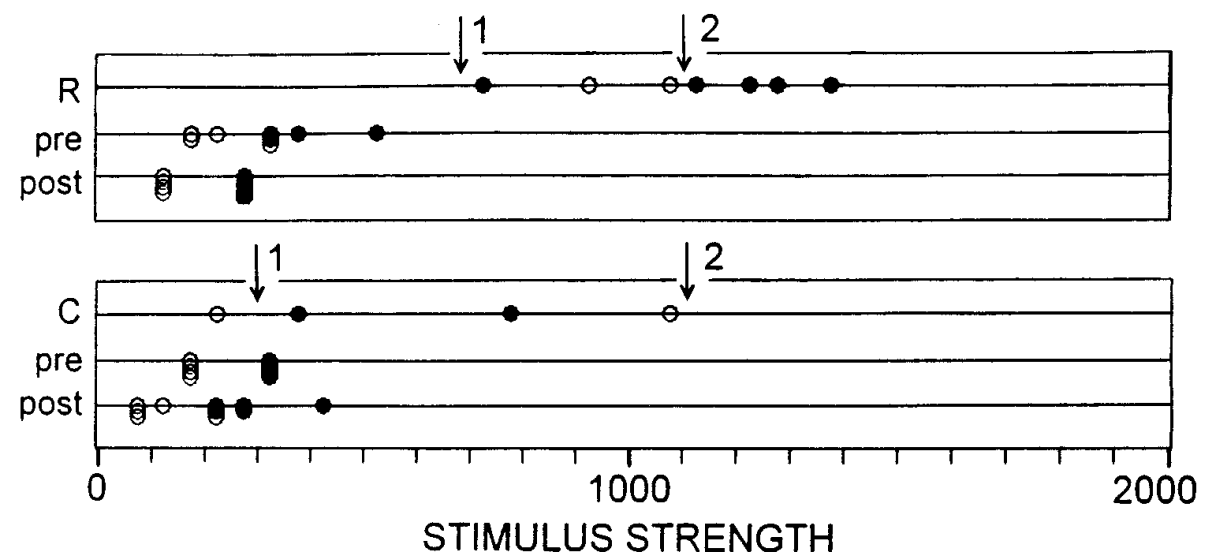



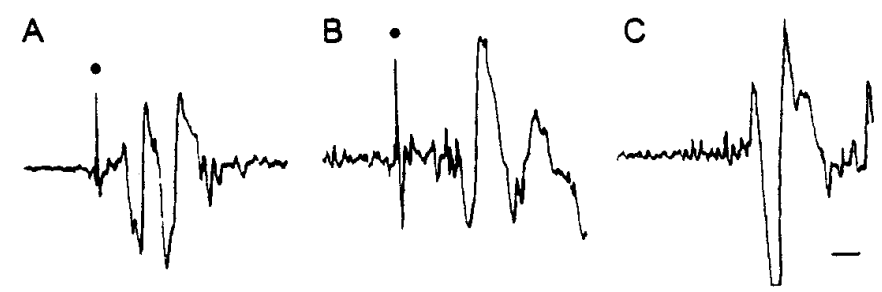

Figure 10. Nongiant escape response of a subordinate during an agonistic encounter. $A$, Lateral giant response evoked by experimenter-produced abdominal tap. $B$, Medial giant response evoked by experimenterproduced tap of cephalothorax. $C$, Response occurring during agonistic encounter. Dots mark giant axon spikes. Slower potentials are phasic flexor muscle potentials. Calibration, $2 \mathrm{msec}$.

\section{Tail flip escape by subordinates during fights is mediated by voluntary, not reflex escape, circuitry}

Although our tests showed the excitability of the LG escape reflex to be reduced in subordinates during social interactions, subordinates typically did escape from their conspecifics by tail flip responses several times per session (although never just at the moment that we were doing a formal behavioral scoring). To try to resolve this possible inconsistency, we recorded electrical activity on tape throughout a number of interaction sessions in six animals so that we could go back and examine the electrical activity associated with each spontaneous tail flip. Figure 10 shows the activity associated with LG and MG tail flips produced by sudden mechanical stimulation of an isolated subordinate and the activity associated with a spontaneous tail flip made by the same animal during interaction with its dominant cohort. It is obvious from these records that the giant axon spikes associated with the experimenter-evoked reflex flips are missing from the spontaneous response, which, thus, was mediated by the nongiant or "voluntary" system. Only 1 of 34 tail flips occurring spontaneously during encounters had giant axon activity preceding it.

\section{DISCUSSION}

This study establishes that the excitability of the LG escape reflex is modulated during agonistic encounters. Furthermore, the pattern of modulation is different in dominants and subordinates. Excitability of LG escape tends to be reduced substantially in subordinates but only slightly so in dominants. It certainly was not expected that dominant animals would be more likely to escape than subordinates, but in retrospect, we believe we can make sense of this observation.

\section{Why excitability of escape might be greater in dominants than subordinates during agonistic interactions}

Lateral and medial giant escape are stereotyped reflexive responses providing fairly effective escape from caudally and rostrally located disturbances, respectively. They occur at very short latency and are thus well suited for escape from stimuli that take an animal by surprise (Wine and Krasne, 1982). For threats that develop more gradually, animals can make nongiant mediated ("voluntary") responses that provide for a much wider range of trajectories and can allow the animal to escape directly away from an obliquely located threat or to swim via a sequence of tail flips into a home burrow, as opposed to simply going "backward" or "upward" relative to previous body position (Wine and Krasne, 1972; Krasne and Wine, 1984). Animals also seem to be able to choose the exact moment of occurrence of these more flexible responses in an adaptive way; animals that are restrained and inhibiting reflexive tail flip responses will often execute nongiant responses at precisely the moment that one slightly loosens hold on them, thereby breaking free of one's grasp (Krasne and Wine, 1975). A variety of lines of evidence indicate that animals that are preparing to make or actually are producing a nongiant escape response inhibit circuitry specifically involved in reflexive responses (Krasne and Wine, 1984). This makes adaptive sense, because the occurrence of a stereotyped reflexive response at such times would be antithetical to the more sophisticated choice and response production strategies of nongiant escape (Kramer and Krasne, 1984). We propose that subordinate animals inhibit reflex escape during agonistic encounters because the wary subordinate commonly is preparing for or standing ready to execute nongiant responses, which are the escape mode of choice for an animal that is not being taken by surprise. Consistent with this proposal, we have seen that escape responses produced by subordinates during encounters are, in fact, mediated by nongiant circuitry.

In contrast to subordinates, dominants usually have little to fear from their conspecifics, need not be wary, and presumably are not preparing continually for nongiant (voluntary) escape, although during periods of intense interaction, they may do so transiently. Thus, in them reflex escape goes relatively uninhibited. Indeed, it could be argued that, because dominants are relying heavily on reflexive escape to protect them from a chance aggressive surge of their partner, it might be adaptive for their escape reflex excitability actually to increase during agonistic encounters, particularly during pauses between periods of more intense interaction. However, we did not see evidence of this here.

\section{Neurochemical mediation of modulation}

Both GABA and, at least in subordinates, serotonin are known to be able to suppress LG escape via direct action on the LGs (Roberts, 1968; Glanzman and Krasne, 1983; Vu and Krasne, 1993; Yeh et al., 1996, 1997). Both octopamine and, at least in dominants, serotonin can increase LG reflex excitability (Glanzman and Krasne, 1983; Yeh et al., 1996, 1997); the octopamine facilitation is known to result in part from action at the synapse between primary afferents and sensory interneurons (Bustamante and Krasne, 1991), but it probably also is mediated by a direct action on the LGs themselves (Lee and Krasne, 1991). The effects observed here easily could be produced by these, or other, agents operating in various combinations. Unfortunately, measurement of amine release within ganglia or into the blood is not straightforward, especially in behaving animals, and antagonists suitable for examining alterations of LG reflex excitability during agonistic behavior in crayfish with particular transmitter systems blocked are not at hand. Picrotoxin can block GABA and phentolamine octopamine action, respectively, but both have nonspecific effects on behavior that almost certainly would interfere with social interactions (our unpublished observations). Furthermore, vertebrate serotonin antagonists that so far have been tried have been without effect on serotonergic modulation of LG excitability $(\mathrm{Vu}$ and Krasne, 1993).

Although proof that serotonin plays a role in crayfish agonistic behavior is lacking, the likelihood of such a role is hinted at by promotion of aggressive stance and increased fighting in crayfish and/or lobsters injected with serotonin (Livingstone et al., 1980; Huber et al., 1995) and by the observation that the effects of serotonin on escape are social status-dependent. In particular, the fact that characteristics of serotonin action seem demonstrably different between dominants and subordinates and between each of these and isolates (Yeh et al., 1996, 1997) suggests that sero- 
tonin is probably released, both in dominants and subordinates, under circumstances when status-specific modulation of the LG escape reflex is required. Obviously, as shown here, agonistic interactions are such a circumstance; indeed, they are the only known such circumstance.

In subordinates both serotonin and periods of interaction reduce the excitability of escape. In dominants both serotonin and periods of interaction have effects different from those seen in subordinates but also somewhat different from each other, for whereas interaction causes a relatively slight and in some cases negligible inhibition of escape, serotonin actually facilitates it. The natural and serotonergic modulations do, however, share the paradox that in both cases modulation causes escape to become much more likely in dominants than in subordinates. Therefore, if serotonin does contribute to the natural modulation, the explanation given above for the behavioral modulation may apply as well to the serotonergic one. The lack of frank facilitation in dominants during interaction could imply that, during natural behavior, facilitatory effects of serotonin are accompanied by other factors that prevent absolute facilitation from occurring. Indeed, our explanation for the differences in modulation between dominants and subordinates does not necessarily predict actual facilitation or even complete lack of inhibition in dominants, because it would be expected that even dominants should be wary under some circumstances, for example during periods of intense sparring with their conspecific; under those circumstances they would be expected to prepare for execution of voluntary escape maneuvers and therefore to inhibit LG (note, e.g., Fig. 8, activities $\mathrm{V}$ and $\mathrm{C}$ in dominants) and presumably also $\mathrm{MG}$ reflex escape. Perhaps on these occasions the putatively GABA-ergic inhibitory system that operates during activities like feeding and restraint (Krasne and Lee, 1988; Vu and Krasne, 1993) overshadows serotonin-caused facilitation.

Whatever the validity of these speculations, the underlying basis for the social status dependency of LG reflex modulation will be an interesting topic for future work.

\section{REFERENCES}

Beall SP, Langley DJ, Edwards DH (1990) Inhibition of escape tail flip in crayfish during backward walking and the defense posture. J Exp Biol 152:577-582.

Bovbjerg RV (1953) Dominance order in the crayfish, Orconectes virilis (Hagen). Physiol Zool 26:173-178.

Bustamante J, Krasne FB (1991) Effects of octopamine on transmission at the first synapse of the crayfish lateral giant escape reaction pathway. $J$ Comp Physiol [A] 169:369-377.
Glanzman DL, Krasne FB (1983) Serotonin and octopamine have opposite modulatory effects on the crayfish's lateral giant escape reaction. J Neurosci 3:2263-2269.

Huber R, Kravitz EA, Helluy S (1995) Serotonin controls fighting behavior in crayfish. Soc Neurosci Abstr 21:1693.

Kennedy D, McVittie J, Calabrese R, Fricke RA, Craelius W, Chiapella P (1980) Inhibition of mechanosensory interneurons in the crayfish. I. Presynaptic inhibition from giant fibers. J Neurophysiol 43:1495-1509.

Kramer AP, Krasne FB (1984) Crayfish escape behavior: production of tail flips without giant fiber activity. J Neurophysiol 52:189-211.

Krasne FB, Bryan JS (1973) Habituation: regulation through presynaptic inhibition. Science 182:590-592.

Krasne FB, Glanzman DL (1986) Sensitization of the crayfish lateral giant escape reaction. J Neurosci 6:1013-1020.

Krasne FB, Lee SC (1988) Response-dedicated trigger neurons as control points for behavioral actions: selective inhibition of lateral giant command neurons during feeding in crayfish. J Neurosci 8:3703-3712.

Krasne FB, Teshiba TM (1995) Habituation of an invertebrate escape reflex due to modulation by higher centers rather than local events. Proc Natl Acad Sci USA 92:3362-3366.

Krasne FB, Wine JJ (1975) Extrinsic modulation of crayfish escape behavior. J Exp Biol 63:433-450.

Krasne FB, Wine JJ (1984) The production of crayfish tail flip escape responses. In: Neural mechanisms of startle behavior (Eaton RC, ed), pp 179-211. New York: Plenum.

Lee SH, Krasne FB (1991) Octopamine increases efficiency of commissural transmission between crayfish lateral giants. Soc Neurosci Abstr 17:1057.

Livingstone MS, Harris-Warrick RM, Kravitz EA (1980) Serotonin and octopamine produce opposite postures in lobsters. Science 208:76-79.

Lowe ME (1956) Dominance-subordinance relationships in Cambarellus shufeldtii. Tulane Stud Zool 4:139-170.

Olson GC, Krasne FB (1981) The crayfish lateral giants as command neurones for escape behaviour. Brain Res 214:89-100.

Roberts AM (1968) Recurrent inhibition in the giant fibre system of the crayfish and its effect on the excitability of the escape response. J Exp Biol 48:545-567.

Vu ET, Krasne FB (1993) Crayfish tonic inhibition: prolonged modulation of behavioral excitability by classical GABA-ergic inhibition. J Neurosci 13:4394-4402.

Vu ET, Lee SC, Krasne FB (1993) The mechanism of tonic inhibition of crayfish escape behavior: distal inhibition and its functional significance. J Neurosci 13:4379-4393.

Wine JJ, Krasne FB (1972) The organization of the escape behavior in the crayfish. J Exp Biol 56:1-18.

Wine JJ, Krasne FB (1982) The cellular organization of crayfish escape behavior. In: The biology of Crustacea, Vol 4 (Sandeman DC, Atwood HL, eds), pp 241-292. New York: Academic.

Yeh SR, Fricke RA, Edwards DH (1996) The effect of social experience on serotonergic modulation of the escape circuit of crayfish. Science 271:366-369.

Yeh SR, Musolf B, Edwards DH (1997) Neuronal adaptations to changes in the social dominance status of crayfish. J Neurosci 17:680-695. 